\title{
MULTIPLE POSITIVE SOLUTIONS OF SINGULAR DISCRETE $p$-LAPLACIAN PROBLEMS VIA VARIATIONAL METHODS
}

RAVI P. AGARWAL, KANISHKA PERERA, AND DONAL O’REGAN

Received 31 March 2005

We obtain multiple positive solutions of singular discrete $p$-Laplacian problems using variational methods.

\section{Introduction}

We consider the boundary value problem

$$
\begin{aligned}
-\Delta\left(\varphi_{p}(\Delta u(k-1))\right) & =f(k, u(k)), \quad k \in[1, n], \\
u(k) & >0, \quad k \in[1, n], \\
u(0) & =0=u(n+1),
\end{aligned}
$$

where $n$ is an integer greater than or equal to $1,[1, n]$ is the discrete interval $\{1, \ldots, n\}$, $\Delta u(k)=u(k+1)-u(k)$ is the forward difference operator, $\varphi_{p}(s)=|s|^{p-2} s, 1<p<\infty$, and we only assume that $f \in C([1, n] \times(0, \infty))$ satisfies

$$
a_{0}(k) \leq f(k, t) \leq a_{1}(k) t^{-\gamma}, \quad(k, t) \in[1, n] \times\left(0, t_{0}\right)
$$

for some nontrivial functions $a_{0}, a_{1} \geq 0$ and $\gamma, t_{0}>0$, so that it may be singular at $t=0$ and may change sign.

Let $\lambda_{1}, \varphi_{1}>0$ be the first eigenvalue and eigenfunction of

$$
\begin{aligned}
-\Delta\left(\varphi_{p}(\Delta u(k-1))\right) & =\lambda \varphi_{p}(u(k)), \quad k \in[1, n], \\
u(0) & =0=u(n+1) .
\end{aligned}
$$

Theorem 1.1. If (1.2) holds and

$$
\limsup _{t \rightarrow \infty} \frac{f(k, t)}{t^{p-1}}<\lambda_{1}, \quad k \in[1, n]
$$

then (1.1) has a solution. 
Theorem 1.2. If (1.2) holds and

$$
f\left(k, t_{1}\right) \leq 0, \quad k \in[1, n]
$$

for some $t_{1}>t_{0}$, then (1.1) has a solution $u_{1}<t_{1}$. If, in addition,

$$
\liminf _{t \rightarrow \infty} \frac{f(k, t)}{t^{p-1}}>\lambda_{1}, \quad k \in[1, n],
$$

then there is a second solution $u_{2}>u_{1}$.

Example 1.3. Problem (1.1) with $f(k, t)=t^{-\gamma}+\lambda t^{\beta}$ has a solution for all $\gamma>0$ and $\lambda$ (resp., $\lambda<\lambda_{1}, \lambda \leq 0$ ) if $\beta<p-1$ (resp., $\beta=p-1, \beta>p-1$ ) by Theorem 1.1.

Example 1.4. Problem (1.1) with $f(k, t)=t^{-\gamma}+e^{t}-\lambda$ has two solutions for all $\gamma>0$ and sufficiently large $\lambda>0$ by Theorem 1.2.

Our results seem new even for $p=2$. Other results on discrete $p$-Laplacian problems can be found in $[1,2]$ in the nonsingular case and in $[3,4,5,6]$ in the singular case.

\section{Preliminaries}

First we recall the weak comparison principle (see, e.g., Jiang et al. [2]).

LEMMA 2.1. If

$$
\begin{gathered}
-\Delta\left(\varphi_{p}(\Delta u(k-1))\right) \geq-\Delta\left(\varphi_{p}(\Delta v(k-1))\right), \quad k \in[1, n], \\
u(0) \geq v(0), \quad u(n+1) \geq v(n+1),
\end{gathered}
$$

then $u \geq v$.

Next we prove a local comparison result.

LEMMA 2.2. If

$$
\begin{gathered}
-\Delta\left(\varphi_{p}(\Delta u(k-1))\right) \geq-\Delta\left(\varphi_{p}(\Delta v(k-1))\right), \\
u(k)=v(k), \quad u(k \pm 1) \geq v(k \pm 1),
\end{gathered}
$$

then $u(k \pm 1)=v(k \pm 1)$.

Proof. We have

$$
\begin{gathered}
-\varphi_{p}(\Delta u(k))+\varphi_{p}(\Delta u(k-1)) \geq-\varphi_{p}(\Delta v(k))+\varphi_{p}(\Delta v(k-1)), \\
\Delta u(k) \geq \Delta v(k), \quad \Delta u(k-1) \leq \Delta v(k-1) .
\end{gathered}
$$

Combining with the strict monotonicity of $\varphi_{p}$ shows that

$$
0 \leq \varphi_{p}(\Delta u(k))-\varphi_{p}(\Delta v(k)) \leq \varphi_{p}(\Delta u(k-1))-\varphi_{p}(\Delta v(k-1)) \leq 0,
$$

and hence, the equalities hold in (2.4). 
The following strong comparison principle is now immediate.

LEMMA 2.3. If

$$
\begin{gathered}
-\Delta\left(\varphi_{p}(\Delta u(k-1))\right) \geq-\Delta\left(\varphi_{p}(\Delta v(k-1))\right), \quad k \in[1, n], \\
u(0) \geq v(0), \quad u(n+1) \geq v(n+1),
\end{gathered}
$$

then either $u>v$ in $[1, n]$, or $u \equiv v$. In particular, if

$$
\begin{gathered}
-\Delta\left(\varphi_{p}(\Delta u(k-1))\right) \geq 0, \quad k \in[1, n], \\
u(0) \geq 0, \quad u(n+1) \geq 0,
\end{gathered}
$$

then either $u>0$ in $[1, n]$ or $u \equiv 0$.

Consider the problem

$$
\begin{aligned}
-\Delta\left(\varphi_{p}(\Delta u(k-1))\right) & =g(k, u(k)), \quad k \in[1, n], \\
u(0)=0 & =u(n+1),
\end{aligned}
$$

where $g \in C([1, n] \times \mathbb{R})$. The class $W$ of functions $u:[0, n+1] \rightarrow \mathbb{R}$ such that $u(0)=0=$ $u(n+1)$ is an $n$-dimensional Banach space under the norm

$$
\|u\|=\left(\sum_{k=1}^{n+1}|\Delta u(k-1)|^{p}\right)^{1 / p}
$$

Define

$$
\Phi_{g}(u)=\sum_{k=1}^{n+1}\left[\frac{1}{p}|\Delta u(k-1)|^{p}-G(k, u(k))\right], \quad u \in W
$$

where $G(k, t)=\int_{0}^{t} g(k, s) d s$. Then the functional $\Phi_{g}$ is $C^{1}$ with

$$
\begin{aligned}
\left(\Phi_{g}^{\prime}(u), v\right) & =\sum_{k=1}^{n+1}\left[\varphi_{p}(\Delta u(k-1)) \Delta v(k-1)-g(k, u(k)) v(k)\right] \\
& =-\sum_{k=1}^{n}\left[\Delta\left(\varphi_{p}(\Delta u(k-1))\right)+g(k, u(k))\right] v(k)
\end{aligned}
$$

(summing by parts), so solutions of (2.8) are precisely the critical points of $\Phi_{g}$.

LEMMA 2.4. If

$$
\limsup _{|t| \rightarrow \infty} \frac{g(k, t)}{|t|^{p-2} t}<\lambda_{1}, \quad k \in[1, n]
$$

then $\Phi_{g}$ has a global minimizer. 
Proof. By (2.12), there is a $\lambda \in\left[0, \lambda_{1}\right)$ such that

$$
G(k, t) \leq \frac{\lambda}{p}|t|^{p}+C
$$

where $C$ denotes a generic positive constant. Since

$$
\lambda_{1}=\min _{u \in W \backslash\{0\}} \frac{\sum_{k=1}^{n+1}|\Delta u(k-1)|^{p}}{\sum_{k=1}^{n}|u(k)|^{p}},
$$

then

$$
\Phi_{g}(u) \geq \frac{1}{p}\left(1-\frac{\lambda}{\lambda_{1}}\right)\|u\|^{p}-C\|u\|,
$$

so $\Phi_{g}$ is bounded from below and coercive.

Lemma 2.5. If

$$
\liminf _{t \rightarrow+\infty} \frac{g(k, t)}{t^{p-1}}>\lambda_{1}, \quad \lim _{t \rightarrow-\infty} \frac{g(k, t)}{|t|^{p-1}}=0, \quad k \in[1, n],
$$

then $\Phi_{g}$ satisfies the Palais-Smale compactness condition $(P S)$ : every sequence $\left(u_{j}\right)$ in $W$ such that $\Phi_{g}\left(u_{j}\right)$ is bounded and $\Phi_{g}^{\prime}\left(u_{j}\right) \rightarrow 0$ has a convergent subsequence.

Proof. It suffices to show that $\left(u_{j}\right)$ is bounded since $W$ is finite dimensional, so suppose that $\rho_{j}:=\left\|u_{j}\right\| \rightarrow \infty$ for some subsequence. We have

$$
o(1)\left\|u_{j}^{-}\right\|=\left(\Phi_{g}^{\prime}\left(u_{j}\right), u_{j}^{-}\right) \leq-\left\|u_{j}^{-}\right\|^{p}-\sum_{k=1}^{n+1} g\left(k,-u_{j}^{-}(k)\right) u_{j}^{-}(k),
$$

where $u_{j}^{-}=\max \left\{-u_{j}, 0\right\}$ is the negative part of $u_{j}$, so it follows from (2.16) that $\left(u_{j}^{-}\right)$is bounded. So, for a further subsequence, $\tilde{u}_{j}:=u_{j} / \rho_{j}$ converges to some $\tilde{u} \geq 0$ in $W$ with $\|\tilde{u}\|=1$.

We may assume that for each $k$, either $\left(u_{j}(k)\right)$ is bounded or $u_{j}(k) \rightarrow \infty$. In the former case, $\tilde{u}(k)=0$ and $g\left(k, u_{j}(k)\right) / \rho_{j}^{p-1} \rightarrow 0$, and in the latter case, $g\left(k, u_{j}(k)\right) \geq 0$ for large $j$ by (2.16). So it follows from

$$
o(1)=\frac{\left(\Phi_{g}^{\prime}\left(u_{j}\right), v\right)}{\rho_{j}^{p-1}}=\sum_{k=1}^{n+1}\left[\varphi_{p}\left(\Delta \tilde{u}_{j}(k-1)\right) \Delta v(k-1)-\frac{g\left(k, u_{j}(k)\right)}{\rho_{j}^{p-1}} v(k)\right]
$$

that

$$
\sum_{k=1}^{n+1} \varphi_{p}(\Delta \tilde{u}(k-1)) \Delta v(k-1) \geq 0 \quad \forall v \geq 0,
$$


and hence, $\tilde{u}>0$ in $[1, n]$ by Lemma 2.3. Then $u_{j}(k) \rightarrow \infty$ for each $k$, and hence, $(2.18)$ can be written as

$$
\sum_{k=1}^{n+1}\left[\varphi_{p}\left(\Delta \tilde{u}_{j}(k-1)\right) \Delta v(k-1)-\alpha_{j}(k) \tilde{u}_{j}(k)^{p-1} v(k)\right]=o(1),
$$

where

$$
\alpha_{j}(k)=\frac{g\left(k, u_{j}(k)\right)}{u_{j}(k)^{p-1}} \geq \lambda, \quad j \text { large }
$$

for some $\lambda>\lambda_{1}$ by (2.16).

Choosing $v$ appropriately and passing to the limit shows that each $\alpha_{j}(k)$ converges to some $\alpha(k) \geq \lambda$ and

$$
\begin{gathered}
-\Delta\left(\varphi_{p}(\Delta \tilde{u}(k-1))\right)=\alpha(k) \tilde{u}(k)^{p-1}, \quad k \in[1, n], \\
\tilde{u}(0)=0=\tilde{u}(n+1) .
\end{gathered}
$$

This implies that the first eigenvalue of the corresponding weighted eigenvalue problem is given by

$$
\min _{u \in W \backslash\{0\}} \frac{\sum_{k=1}^{n+1}|\Delta u(k-1)|^{p}}{\sum_{k=1}^{n} \alpha(k)|u(k)|^{p}}=1 .
$$

Then

$$
1 \leq \frac{\sum_{k=1}^{n+1}\left|\Delta \varphi_{1}(k-1)\right|^{p}}{\sum_{k=1}^{n} \alpha(k) \varphi_{1}(k)^{p}} \leq \frac{\lambda_{1}}{\lambda}<1,
$$

a contradiction.

\section{Proofs}

The problem

$$
\begin{gathered}
-\Delta\left(\varphi_{p}(\Delta u(k-1))\right)=a_{0}(k), \quad k \in[1, n], \\
u(0)=0=u(n+1),
\end{gathered}
$$

has a unique solution $u_{0}>0$ by Lemmas 2.3 and 2.4 . Fix $\varepsilon \in(0,1]$ so small that $\underline{u}:=$ $\varepsilon^{1 /(p-1)} u_{0}<t_{0}$. Then

$$
-\Delta\left(\varphi_{p}(\Delta \underline{u}(k-1))\right)-f(k, \underline{u}(k)) \leq-(1-\varepsilon) a_{0}(k) \leq 0
$$

by (1.2), so $\underline{u}$ is a subsolution of (1.1). Let

$$
f_{\underline{u}}(k, t)= \begin{cases}f(k, t), & t \geq \underline{u}(k), \\ f(k, \underline{u}(k)), & t<\underline{u}(k) .\end{cases}
$$


Proof of Theorem 1.1. By (1.4), there are $\lambda \in\left[0, \lambda_{1}\right)$ and $T>t_{0}$ such that

$$
f(k, t) \leq \lambda t^{p-1}, \quad(k, t) \in[1, n] \times(T, \infty) .
$$

Then

$$
f_{\underline{u}}(k, t) \begin{cases}\leq a_{1}(k) \underline{u}(k)^{-\gamma}+\max f\left([1, n] \times\left[t_{0}, T\right]\right)+\lambda t^{p-1}, & t \geq 0, \\ \geq a_{0}(k), & t<0,\end{cases}
$$

by (1.2), so the modified problem

$$
\begin{gathered}
-\Delta\left(\varphi_{p}(\Delta u(k-1))\right)=f_{\underline{u}}(k, u(k)), \quad k \in[1, n], \\
u(0)=0=u(n+1),
\end{gathered}
$$

has a solution $u$ by Lemma 2.4. By Lemma $2.1, u \geq \underline{u}$, and hence, also a solution of (1.1).

Proof of Theorem 1.2. Noting that $t_{1}$ is a supersolution of (3.6), let

$$
\tilde{f}_{\underline{u}}(k, t)= \begin{cases}f_{\underline{u}}\left(k, t_{1}\right), & t>t_{1}, \\ f_{\underline{u}}(k, t), & t \leq t_{1} .\end{cases}
$$

By (1.2),

$$
\tilde{f}_{\underline{u}}(k, t) \begin{cases}\leq a_{1}(k) \underline{u}(k)^{-\gamma}+\max f\left([1, n] \times\left[t_{0}, t_{1}\right]\right), & t \geq 0, \\ \geq a_{0}(k), & t<0,\end{cases}
$$

so $\Phi_{\widetilde{f}_{\underline{u}}}$ has a global minimizer $u_{1}$ by Lemma 2.4. By Lemmas 2.1 and $2.2, \underline{u} \leq u_{1}<t_{1}$, so $\Phi_{\widetilde{f}_{\underline{u}}} \stackrel{\stackrel{\underline{u}}{=}}{=} \Phi_{f_{\underline{u}}}$ near $u_{1}$ and hence, $u_{1}$ is a local minimizer of $\Phi_{f_{\underline{u}}}$. Let

$$
f_{u_{1}}(k, t)= \begin{cases}f(k, t), & t \geq u_{1}(k), \\ f\left(k, u_{1}(k)\right), & t<u_{1}(k)\end{cases}
$$

Since $u_{1}$ is also a subsolution of (1.1), repeating the above argument with $u_{1}$ in place of $\underline{u}$, we see that $\Phi_{f_{u_{1}}}$ also has a local minimizer, which we assume is $u_{1}$ itself, for otherwise we are done. By (1.6), there are $\lambda>\lambda_{1}$ and $T>t_{1}$ such that

$$
f(k, t) \geq \lambda t^{p-1}, \quad(k, t) \in[1, n] \times(T, \infty),
$$

so

$$
\Phi_{f_{u_{1}}}\left(t \varphi_{1}\right) \leq-\frac{t^{p}}{p}\left(\frac{\lambda}{\lambda_{1}}-1\right)+C t<\Phi_{f_{u_{1}}}\left(u_{1}\right), \quad t>0 \text { large. }
$$

Since $\Phi_{f_{u_{1}}}$ satisfies (PS) by Lemma 2.5, the mountain-pass lemma now gives a second critical point $u_{2}$, which is greater than $u_{1}$ by Lemmas 2.1 and 2.2. 


\section{References}

[1] R. Avery and J. Henderson, Existence of three positive pseudo-symmetric solutions for a one dimensional discrete p-Laplacian, J. Difference Equ. Appl. 10 (2004), no. 6, 529-539.

[2] D. Jiang, J. Chu, D. O'Regan, and R. P. Agarwal, Positive solutions for continuous and discrete boundary value problems to the one-dimension p-Laplacian, Math. Inequal. Appl. 7 (2004), no. $4,523-534$.

[3] D. Jiang, D. O'Regan, and R. P. Agarwal, A generalized upper and lower solution method for singular discrete boundary value problems for the one-dimensional p-Laplacian, to appear in J. Appl. Anal.

[4] Existence theory for single and multiple solutions to singular boundary value problems for the one-dimension p-Laplacian, Adv. Math. Sci. Appl. 13 (2003), no. 1, 179-199.

[5] D. Jiang, L. Zhang, D. O'Regan, and R. P. Agarwal, Existence theory for single and multiple solutions to singular positone discrete Dirichlet boundary value problems to the one-dimension p-Laplacian, Archivum Mathematicum (Brno) 40 (2004), no. 4, 367-381.

[6] D. Q. Jiang, P. Y. H. Pang, and R. P. Agarwal, Upper and lower solutions method and a superlinear singular discrete boundary value problem, to appear in Dynam. Systems Appl.

Ravi P. Agarwal: Department of Mathematical Sciences, Florida Institute of Technology, Melbourne, FL 32901, USA

E-mail address: agarwal@fit.edu

Kanishka Perera: Department of Mathematical Sciences, Florida Institute of Technology, Melbourne, FL 32901, USA

E-mail address: kperera@fit.edu

Donal O'Regan: Department of Mathematics, National University of Ireland, Galway, Ireland

E-mail address: donal.oregan@nuigalway.ie 